\title{
Buck-Boost Converter with Fractional Control for Electric Vehicles
}

\author{
Rubén Martínez ${ }^{1}$, Yolanda Bolea ${ }^{1}$, Antoni Grau ${ }^{1}$ and Herminio Martínez ${ }^{2}$ \\ ${ }^{1}$ Automatic Control Dept \\ Technical University of Catalonia \\ Barcelona, Spain. \\ e-mail: \{ ruben.martinez.gonzalez, yolanda.bolea,antoni.grau\}@upc.edu \\ ${ }^{2}$ Electronics Engineering Dept \\ Technical University of Catalonia \\ Barcelona, Spain \\ e-mail: herminio.martinez@upc.edu
}

\begin{abstract}
In this paper, non-integer order control is applied to improve the performance of a buck-boost dc-dc power converter with ultracapacitor based on state feedback techniques. The approach is based on stabilization in the Lyapunov's sense for linear rational-order systems. The ultracapacitor can overcome the classical problems of energy losses due to the use of batteries, giving more accuracy, autonomy and efficiency to the electrical vehicles.
\end{abstract}

\section{Key words}

Electrical vehicles, buck-boost converter, ultracapacitor, fractional control, energy saving.

\section{Introduction}

With the decrease of fossil fuel reserves, there is an increased demand for hybrid vehicles. Hybrid vehicles under development provide cheap, dependable and clean improvements in fuel economy. Essentially, all hybrid electric vehicles including fuel cell vehicles require energy storage systems, commonly battery packs.

Problems exist with battery packs including the inability to absorb and discharge large current loads during regenerative braking and boost assist, performance degradation over their life, weight, size and environmental concerns regarding disposal.

Ultracapacitors can eliminate these problems. Moreover, the use of DC-DC switching converter is mandatory in this kind of applications [1].

In fact, the advantages of DC-DC switching converters are evident opposite to other kind of DC-DC converters like, for instance, linear regulators: the efficiency of them, in spite of not arriving at the $100 \%$ due to the omnipresent circuit losses, is near to this optimal value. In addition, there is a wide variety of structures such as step-down (buck) converters, step-up (boost) converters and buck-boost converters.

However, notice that switching converters show some important problems that restrict the general applications in many power supply systems (for example, the design and implementation of this sort of converters is a more complex process than in other DC-DC converters such as, for instance, linear regulators, especially their control loops when both line and load regulations are desired; the intrinsic switched nature of these converters produces ripples in the output voltage and an increment of the EMIs in neighboring electronic systems, etc.).

Ultracapacitors are storage devices which store the energy within the electrochemical double layer at the electrode/electrolyte interface.

Usually, the ultracapacitors are modelled with a semiinfinite RC transmission line with losses this model can describe the behaviour of the ultracapacitor at low or high frequencies, but this model is not valid when the frequency range is in medium frequencies. This zone is characterized by diffusion effect, and according to some papers is better characterized in the Warburg domain $(j w)^{1 / 2}$ [2] than in the classical Laplace domain (jw). In other words, ultracapacitors are characterized by noninteger order differential equations according to the frequency range of operation. Figure 1 displays Nyquist diagram of both devices.

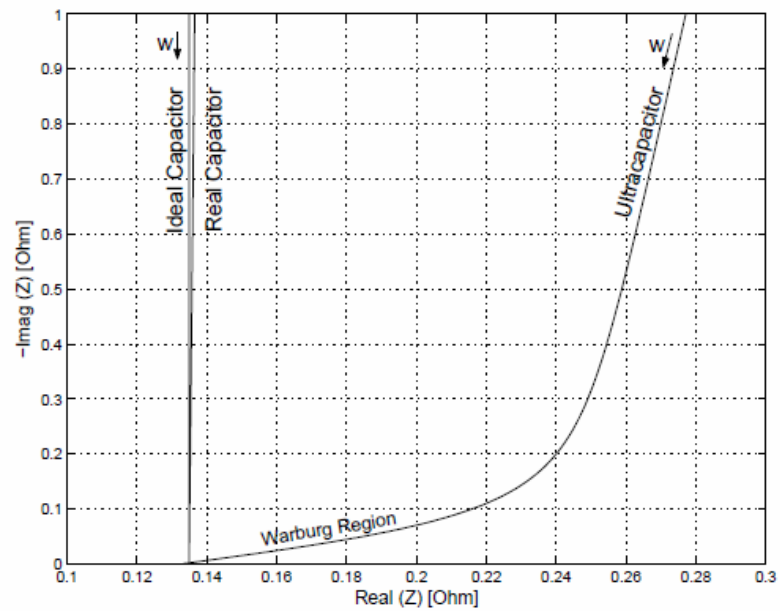

Fig. 1. Nyquist diagram of a capacitor (real and ideal) and an ultracapacitor.. 
The idea of non-integer order derivatives is not new, in the last years many authors [4] have modelled and controlled many physical phenomena based in fractional calculus. This mathematical phenomenon allows to describe a real object more accurately than the classical integer methods.

Real objects are generally of fractional order; however, for many of them the fractionality is very low.

The control systems used so far were all considered as integer-order systems, regardless of the reality. But these works have usually theoretical character, whereas the number of works in which a real object is analyzed and a rational-order controller is designed and implemented is small. The main reason for this fact, taking into account the theoretical advantages of fractional controllers for some control problems, is the difficulty of controller implementation.

In this paper, non-integer order control is applied to improve the performance of a buck-boost DC-DC power converter with ultracapacitor-based on state feedback techniques.

The buck-boost converter is a type of DC-DC converter that has an output voltage magnitude that is either greater than or less than the input voltage magnitude. This converter is conceived to be used as a controlled energytransfer-equipment between the main energy source of an electric vehicle (a battery pack in this case) and an auxiliary energy system based on ultracapacitors.

In all switching converters, the output voltage is a function of the input line voltage, the duty cycle and the load current, as well as the converter circuit element values [1]. In a DC-DC converter application, it is desired to obtain a constant output voltage in spite of disturbances in the input voltage and load current, and in spite of variations in the converter circuit element values. Note that the sources of these disturbances and variations can be many (periodic variations of the input voltage produced by rectifier circuits, significant variations of the load, etc.).

Therefore, we cannot expect to simply set the DC-DC converter duty cycle to a single value, and obtain a given constant output voltage under all work conditions. Then, a negative feedback control loop is mandatory in order to obtain a circuit that automatically adjusts the duty cycle as necessary to achieve the desired output voltage with high accuracy, regardless of disturbances in the input voltage and output current or variations in component values.

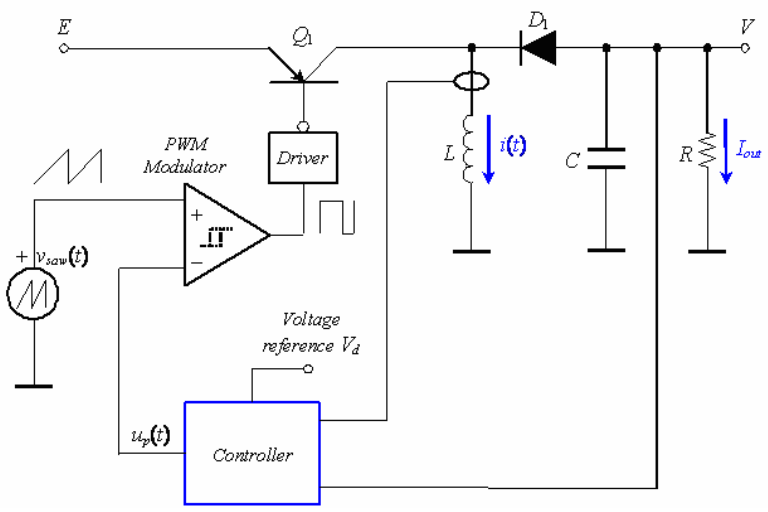

Fig. 2. General structure of a DC-DC buck-boost converter.
A typical block diagram of the feedback system for a buck-boost converter is shown in Fig. 2. The output voltage $V$ and the inductor current $i(t)$ are measured using sensor devices. These measured signals are applied to the controller block in order to achieve the control law $u_{p}(t)$ at its output. This control signal is applied to the pulsewidth modulator block that produces a binary signal that commands the converter power transistor $Q_{1}$ to switch on and off. The function of the pulse-width modulator is to produce a duty ratio that is proportional to the analog control voltage $u_{p}(t)$, achieving the desired output voltage $V=V_{d}$.

Let us consider the tangent linearization model of the average normalized Buck-Boost converter system, whose impedance of the ultracapacitor includes an additional parameter $\theta$,

$$
Z_{C}=\frac{1}{(j w)^{\theta} C}
$$

where $C$ is constant and represents the capacitance. So, for these conditions, the system can be defined by equations:

$$
\begin{aligned}
& D_{\tau}^{\sigma} x_{1}=u_{p} x_{2}+\left(1-u_{p}\right) \\
& D_{\tau}^{\theta} x_{2}=-u_{p} x_{1}-\frac{1}{Q} x_{2}
\end{aligned}
$$

where the variable $x_{1}$ represents the normalized inductor current, $x_{2}$ is the normalized output voltage and $u_{p}$ represents the average control variable.

Clearly the underlying transformation is given by:

$$
\left[\begin{array}{l}
x_{1} \\
x_{2}
\end{array}\right]=\left[\begin{array}{cc}
\frac{1}{E} \sqrt{\frac{L}{C}} & 0 \\
0 & \frac{1}{E}
\end{array}\right]\left[\begin{array}{c}
i(t) \\
v(t)
\end{array}\right], Q=R \sqrt{C / L}
$$

and

$$
\tau=t / \sqrt{C / L}
$$

around the equilibrium point:

$$
x_{2}^{e}=V_{d}<0, x_{1}^{e}=-\left(1-V_{d}\right) \frac{V_{d}}{Q} \text { and } u_{p}^{e}=\frac{1}{1-V_{d}}
$$

where $V_{d}$ is the normalized reference voltage, $v(t)$ is the output voltage, $i(t)$ is the output current, $E, R, L$ and $C$ are the parameters of the passive components that present the circuit.

So, in this paper, an average linear state feedback controller will be designed, driving the average stabilization error state to zero in a generalized exponentially stable fashion. The approach is based on stabilization in the Lyapunov's sense for linear rational order systems.

\section{A Survey of Fractional Calculus}

The idea of non-integer order derivates is as old as regular calculus. Fractional calculus has been used for modelling different physical phenomena [3] and in control theory ([4]; [5]; [6]). We can notice systems in nature with fractional behaviour, but many of them with a very low fractionality [7]. 
The fractional integral operator is defined by [3]

$$
I_{t}^{\alpha} f(t)=\frac{1}{\Gamma(\alpha)} \int_{0}^{t}(t-\tau)^{\alpha-1} f(\tau) d \tau
$$

and we adopt the Caputo definition for fractional derivative of order $\alpha$ of any function $f(t)$ :

$$
D_{t}^{\alpha} f(t)=\frac{1}{\Gamma(n-\alpha)} \int_{0}^{t}(t-\tau)^{n-\alpha-1} f^{(n)}(\tau) d \tau
$$

where $n-1<\alpha<n, \alpha \in \mathfrak{R}^{+}$and the gamma function $\Gamma(v)$ is defined for $v>0$ as:

$$
\Gamma(v)=\int_{0}^{\infty} x^{v-1} e^{-x} d x
$$

\section{State Feedback Controller Design}

The ultracapacitors can be modelling by zones. At low and higher frequencies is similar to a classical capacitor $(\alpha=1)$ and at medium frequencies is characterized by diffusion effect and is better characterized in the Warburg domain $(\alpha=0.5)$. The impedance of the inductor is considered as ideal $(\sigma=1)$.

Let us consider the tangent linearization model of the average normalized ideal Buck-Boost converter system defined by (1) whose linearization of the average model is given by

$$
\begin{gathered}
D_{\tau}^{1} e_{1}=\frac{1}{1-V_{d}} e_{2}-\left(1-V_{d}\right) u_{e} \\
D_{\tau}^{\alpha(w)} e_{2}=-\frac{1}{1-V_{d}} e_{1}-\frac{1}{Q} e 2+\frac{V_{d}\left(1-V_{d}\right)}{Q} u_{e}
\end{gathered}
$$

where $e_{1}=x_{1}-x_{1}^{e}, e_{2}=x_{2}-x_{2}^{e}$ and $u_{e}=u_{p}-u_{p}^{e}$.

The objective is to find an stabilizing control law $u_{e}(t)$ such as:

1) The equilibrium point $e=0$ of (5) - (6) is locally and asymptotically stable.

2) The control system must reject constant disturbances.

3) The eigenvalues of the average feedback state can be arbitrarily assigned.

Because ultracapacitors can be modelled by zones, integer and non-integer order controls are proposed. The response of the system is compared for each case in the simulation results.

\section{A. Integer Order (IO) Controller.}

In this case, it seeks an average integer and linear state feedback control of the form:

$$
u_{e}=-k_{1} I_{\tau}^{1} e_{1}-k_{2} e_{1}-k_{3} e_{2}
$$

which drives the average stabilization error state $e$ to zero in an exponentially stable fashion. It design such a controller with the help of the average tangent linearization system and will use, for the average nonlinear system, the control input:

$$
u_{p}=u_{p}^{e}-k_{1} I_{\tau}^{1} e_{1}-k_{2} e_{1}-k_{3} e_{2}
$$

At low and higher frequencies $(\alpha=1)$, the equivalent closed loop tangent system is given by:

$$
\begin{gathered}
D_{\tau}^{2} e_{1}=H k_{2} D_{\tau}^{1} e_{1}+H k_{1} e_{1}+\left(H k_{3}+1 / H\right) D_{\tau}^{1} e_{2} \\
D_{\tau}^{2} e_{2}=-\left(P k_{3}+\frac{1}{Q}\right) D_{\tau}^{1} e_{2}-\left(P k_{2}+\frac{1}{H}\right) D_{\tau}^{1} e_{1}-P k_{1} e_{1} \text { wh } \\
\text { ere } P=\frac{V_{d} H}{Q} \text { and } H=1-V_{d} . \text { In matrix form is }
\end{gathered}
$$
expressed as:

$$
D_{\tau}^{1} e_{i o i o}=\left[\begin{array}{ccc}
0 & 1 & 0 \\
h_{1} & h_{2} & h_{3} \\
h_{4} & h_{5} & h_{6}
\end{array}\right] e_{\text {ioio }}
$$

where

$$
\begin{aligned}
& e_{\text {ioio }}=\left[\begin{array}{lll}
e_{1} & D_{\tau}^{1} e_{1} & D_{\tau}^{1} e_{2}
\end{array}\right]^{T}, h_{1}=H k_{1}, h_{2}=H k_{2}, \\
& h_{3}=H k_{3}+\frac{1}{H}, h_{4}=-P k_{1} \\
& h_{5}=-\left(P k_{2}+\frac{1}{H}\right), h_{6}=-\left(P k_{3}+\frac{1}{Q}\right)
\end{aligned}
$$

and whose characteristic polynomial is given by

for

$$
P(s)=s^{3}+a_{1 i o} s^{2}+a_{2 i o} s+a_{3 i o}
$$

$$
\begin{aligned}
& a_{1 i o}=P k_{3}-H k_{2}+\frac{1}{Q} \\
& a_{2 i o}=k_{3}+H^{-2}+\frac{\left(2 V_{d}-1\right)}{Q} k_{2}-H k_{1} \\
& a_{3 i o}=\frac{\left(2 V_{d}-1\right)}{Q} k_{1}
\end{aligned}
$$

For medium frequencies $(\alpha=0.5)$, the equivalent closed loop tangent system is given by:

$$
\begin{gathered}
D_{\tau}^{2} e_{1}=H k_{2} D_{\tau}^{1} e_{1}+H k_{1} e_{1}+\left(H k_{3}+1 / H\right) D_{\tau}^{1} e_{2} \\
D_{\tau}^{1.5} e_{2}=-\left(P k_{3}+\frac{1}{Q}\right) D_{\tau}^{1} e_{2}-\left(P k_{2}+\frac{1}{H}\right) D_{\tau}^{1} e_{1}-P k_{1} e_{1}
\end{gathered}
$$

In matrix form is expressed as:

$$
D_{\tau}^{0.5} e_{\text {iofo }}=\left[\begin{array}{ccccc}
0 & 1 & 0 & 0 & 0 \\
0 & 0 & 1 & 0 & 0 \\
0 & 0 & 0 & 1 & 0 \\
h_{1} & 0 & h_{21} & 0 & h_{3} \\
h_{4} & 0 & h_{5} & 0 & h_{6}
\end{array}\right] e_{i o f o}
$$

where $e_{\text {iofo }}=\left[\begin{array}{lllll}e_{1} & D_{\tau}^{0.5} e_{1} & D_{\tau}^{1} e_{1} & D_{\tau}^{1.5} e_{1} & D_{\tau}^{1} e_{2}\end{array}\right]^{T}$ and whose characteristic polynomial is given by

$$
P(\lambda)=\lambda^{5}+a_{1 f o} \lambda^{4}+a_{2 f o} \lambda^{3}+a_{3 f o} \lambda^{2}+a_{4 f o} \lambda+a_{5 f o}
$$

for $\lambda=s^{0.5}$ and

$a_{1 f o}=P k_{3}+\frac{1}{Q}, a_{2 f o}=-H k_{2}, a_{4 f o}=-H k_{1}$

$a_{3 f o}=k_{3}+\frac{\left(2 V_{d}-1\right)}{Q} k_{2}+H^{-2}, a_{5 f o}=\frac{\left(2 V_{d}-1\right)}{Q} k_{1}$ 


\section{B. Non-Integer Order (FO) Controller.}

In this section, the degree of freedom afforded by fractional models in state space is used to offer fractional controllers for each plant.

For low and higher frequencies, it seeks an average integer and linear state feedback control of the form:

$$
u_{e}=-q_{1} I_{\tau}^{0.5} e_{1}-q_{2} e_{1}-q_{3} D_{\tau}^{0.5} e_{1}-q_{4} e_{2}-q_{5} D_{\tau}^{0.5} e_{2}
$$

which drives the average stabilization error state $e$ to zero in an exponentially stable fashion. It designs such a controller with the help of the average tangent linearization system and will use, for the average nonlinear system, the control input:

$$
u_{p}=u_{p}^{e}+u_{e}
$$

The equivalent closed loop tangent system is given by:

$$
\begin{gathered}
D_{\tau}^{1.5} e_{1}=H q_{1} e_{1}+H q_{2} D_{\tau}^{0.5} e_{1}+H q_{3} D_{\tau}^{1} e_{1}+ \\
\left(\frac{1}{H}+H q_{4}\right) D_{\tau}^{0.5} e_{2}+H q_{5} D_{\tau}^{1} e_{2} \\
D_{\tau}^{1.5} e_{2}=-P q_{1} e_{1}-\left(P q_{2}+\frac{1}{H}\right) D_{\tau}^{0.5} e_{1}-P q_{3} D_{\tau}^{1} e_{1}- \\
\left(\frac{1}{Q}+P q_{4}\right) D_{\tau}^{0.5} e_{2}-P q_{5} D_{\tau}^{1} e_{2}
\end{gathered}
$$

In matrix form is expressed as:

$$
D_{\tau}^{0.5} e_{\text {foio }}=\left[\begin{array}{ccccc}
0 & 1 & 0 & 0 & 0 \\
0 & 0 & 1 & 0 & 0 \\
g_{1} & g_{2} & g_{3} & g_{4} & g_{5} \\
0 & 0 & 0 & 0 & 1 \\
g_{6} & g_{7} & g_{8} & g_{9} & g_{10}
\end{array}\right] e_{f o i o}
$$

where

$e_{\text {foio }}=\left[\begin{array}{lllll}e_{1} & D_{\tau}^{0.5} e_{1} & D_{\tau}^{1} e_{1} & D_{\tau}^{0.5} e_{2} & D_{\tau}^{1} e_{2}\end{array}\right]^{T}, \quad g_{1}=H q_{1}$,

$g_{2}=H q_{2}, g_{3}=H q_{3}, g_{5}=H q_{5}$

$g_{4}=H q_{4}+\frac{1}{H}, g_{6}=-P q_{1}, g_{8}=-P q_{3}$

$g_{7}=-\left(P q_{2}+\frac{1}{H}\right), g_{9}=-\left(P q_{4}+\frac{1}{Q}\right)$

$g_{10}=-P q_{5}$

and whose characteristic polynomial is given by

for

$$
P(\lambda)=\lambda^{5}+b_{1 i o} \lambda^{4}+b_{2 i o} \lambda^{3}+b_{3 i o} \lambda^{2}+b_{4 i o} \lambda+b_{5 i o}
$$

$b_{1 i o}=P q_{5}-H q_{3}, b_{1 i o}=P q_{4}-H q_{2}+\frac{1}{Q}$

$b_{3 i o}=q_{5}+\frac{\left(2 V_{d}-1\right)}{Q} q_{3}-H q_{1}$

$b_{4 i o}=q_{4}+\frac{\left(2 V_{d}-1\right)}{Q} q_{2}+H^{-2}$

$b_{5 i o}=\frac{\left(2 V_{d}-1\right)}{Q} q_{1}$
For medium frequencies $(\alpha=0.5)$, it seeks an average non-integer and linear state feedback control of the form:

$$
u_{e}=-q_{1} I_{\tau}^{0.5} e_{1}-q_{2} e_{1}-q_{3} D_{\tau}^{0.5} e_{1}-q_{4} e_{2}
$$

The equivalent closed loop tangent system is given by:

$$
\begin{gathered}
D_{\tau}^{1.5} e_{1}=H q_{1} e_{1}+H q_{2} D_{\tau}^{0.5} e_{1}+H q_{3} D_{\tau}^{1} e_{1}+ \\
\left(\frac{1}{H}+H q_{4}\right) D_{\tau}^{0.5} e_{2} \\
D_{\tau}^{1} e_{2}=-P q_{1} e_{1}-\left(P q_{2}+\frac{1}{H}\right) D_{\tau}^{0.5} e_{1}-P q_{3} D_{\tau}^{1} e_{1}- \\
\left(\frac{1}{Q}+P q_{4}\right) D_{\tau}^{0.5} e_{2}
\end{gathered}
$$

In matrix form is expressed as:

$$
D_{\tau}^{0.5} e_{\text {foio }}=\left[\begin{array}{cccc}
0 & 1 & 0 & 0 \\
0 & 0 & 1 & 0 \\
g_{1} & g_{2} & g_{3} & g_{4} \\
g_{6} & g_{7} & g_{8} & g_{9}
\end{array}\right] e_{f o f o}
$$

where $e_{\text {fofo }}=\left[\begin{array}{llll}e_{1} & D_{\tau}^{0.5} e_{1} & D_{\tau}^{1} e_{1} & D_{\tau}^{0.5} e_{2}\end{array}\right]^{T}$

and whose characteristic polynomial is given by

$$
P(\lambda)=\lambda^{4}+b_{1 f o} \lambda^{3}+b_{2 f_{o}} \lambda^{2}+b_{3 f o} \lambda+a_{4 f o}
$$

for $\lambda=s^{0.5}$ and

$b_{1 f o}=P q_{4}-H q_{3}+\frac{1}{Q}$,

$b_{2 f o}=\frac{2 V_{d}-1}{Q} q_{3}-H q_{2}$,

$a_{3 f o}=q_{4}+\frac{\left(2 V_{d}-1\right)}{Q} q_{2}+H^{-2}-H q_{1}$,

$a_{4 f o}=\frac{\left(2 V_{d}-1\right)}{Q} q_{1}$

Equating these polynomials to a desired closed loop characteristic polynomial can be obtained the feedback gains for the rational linear controllers.

\section{Simulations}

Simulations are performed to assess the effectiveness of the proposed full state feedback controllers, computed on basis of the tangent linearized systems, to accomplish a stabilization around a normalized equilibrium point value for initial conditions set at origin of coordinates.

In order to compare the performances of the different control laws (IO and FO controllers for IO and FO plants), the same poles placement to closed loop system is used for determinate the feedback gains. All roots of the characteristic polynomial are defined by $\zeta$.

The following parameters and design values are used:

$$
Q=0.75, E=10 \mathrm{~V}, V_{d}=-5 \mathrm{~V}, \zeta=0.15
$$




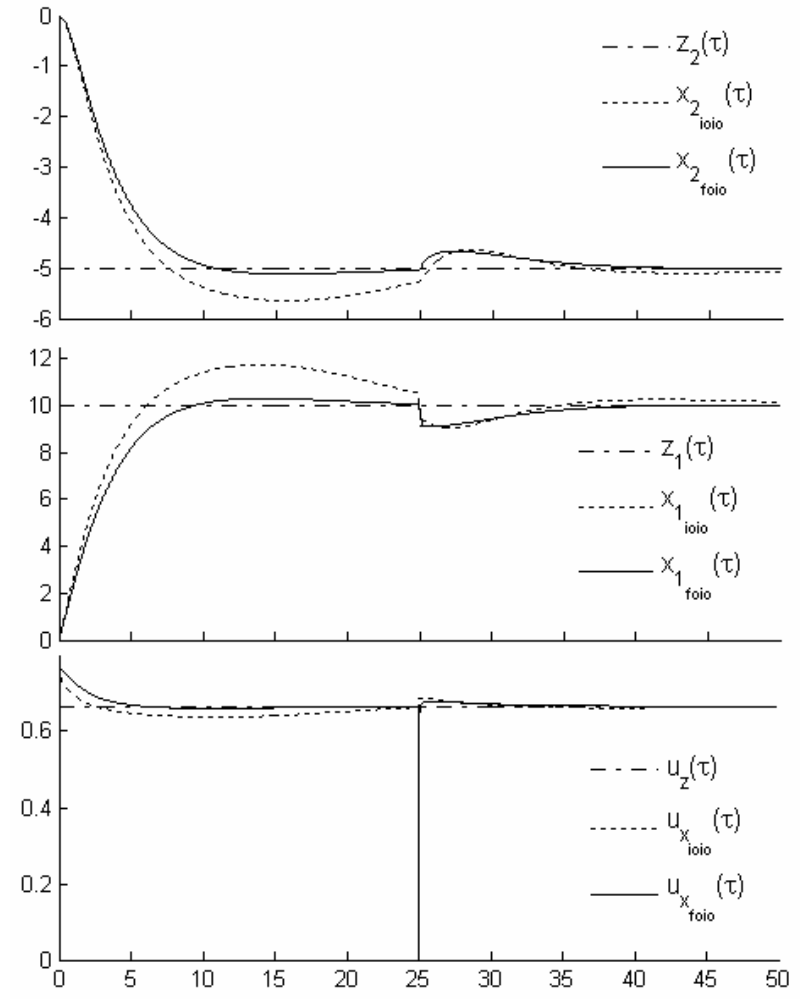

Fig. 3. Response of average Buck-Boost converter based on ultracapacitors to linear state feedback controllers $(\alpha=1)$.

An slower response is proposed because values of $\zeta$ that demand faster responses the average control input initially take negative values. This would cause a temporary saturation to zero of the corresponding switched controller. In order to verify that the control system rejects constant disturbances, at $t=25 \mathrm{~s}$ is used a step signal as disturbance on output. Fig. 3 depicts the response of the of nonlinear average Buck-Boost converter circuit based on ultracapacitors for low frequencies to $\mathrm{IO}$ and $\mathrm{FO}$ control actions of state feedback controllers computed on the basis of the linearized tangent average system complemented with the nominal equilibrium control input.

Similarly, Fig. 4 depicts the response of the nonlinear average Buck-Boost converter circuit for medium frequencies. In both plants (IO and FO plants), FO controllers show a best behaviour at closed loop system. The responses are softer and the convergence to the origin is higher.

\section{Conclusions}

In this work, fractional modelling of a DC-DC buckboost converter based on ultracapacitors, suitable for many powered electrical systems, is presented. As the fractional model of the system changes according to the frequencies range, FO and IO models are proposed. Therefore, FO and IO linear feedback controllers are designed and compared in each plant. In simulation results is showed that FO controllers are more suitable for both plants (IO and FO plants) than IO controllers, which represents a strong motivation to the modelling and control of powered electrical systems via fractional control techniques.

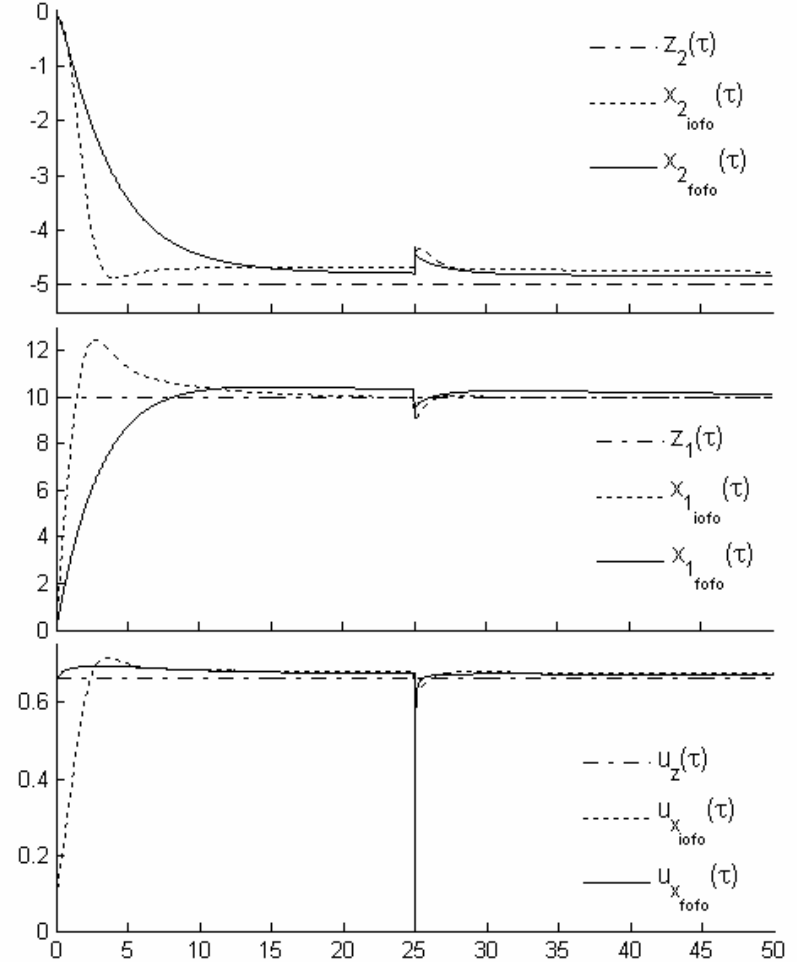

Fig. 4. Response of average Buck-Boost converter based on ultracapacitors to linear state feedback controllers $(\alpha=0.5)$.

\section{Acknowledgement}

Authors would like to thank Inter-Ministerial Commission of Spanish Government (DPI2004-5414, MCYT), Catalan Autonomous Government (VIS, Consolidated Research Group), European Commission (FP6-2005-IST-6, URUS-045062) and Cooperation Spanish Agency for International Development (AECID) for funding this research.

\section{References}

[1] Erickson, R. W. and Maksimovic, D. "Fundamentals of Power Electronics", $2^{\text {nd }}$ edition, Ed. Kluwer Academic Publishers, 2001.

[2] Pintelon, R., "Identification of linear time invariant difusion phenomena", IEEE Trans. on Instrumentation and Measurement, vol. 47, no. 5, October 1998.

[3] Podlubny, I., "Fractional Differential equations", San Diego: Academic Press, 1999.

[4] Podlubny, I., "Fractional-order systems and $\mathrm{PI}^{\rho} \mathrm{D}^{\mu}$ controllers", IEEE Trans. on Automatic Control, vol. 44, no. 1 , pp. $208-214,1999$.

[5] Tenreiro Machado, J. A., "Analysis and Design of Fractional-Order Digital Control Systems", Journal Systems Analysis-Modelling-Simulation, Gordon \& Breach Science Publishers, vol. 27,pp. 107 - 122, 1997.

[6] Lanusse, P., Oustaloup, A. and Sabatier, J., "Step-By-Step Presentation of a 3rd Generation CRONE Controller Design With An Anti-Windup System", ENOC-2005, EUROMECH Nonlinear Oscillations Conference, Eindhoven, Netherlands, 2005.

[7] Torvik, P.J. and Bagley, R. L., "On the Appearance of the Fractional Derivative in the Behavior of Real Materials", Transactions of the ASME, 51, 294 - 298, 1984. 